\title{
Prostate Cancer Highlights from ASCO GU 2021: Cutting-Edge Results
}

\author{
Alexander Meisel \\ Associate Researcher \\ University Hospital Zurich \\ Zurich, Switzerland
}

\section{DOI: 10.36000/HBT.OH.2021.08.041}

Meisel A. Prostate Cancer Highlights from ASCO GU 2021: Cutting-Edge Results. healthbook TIMES Onco Hema. 2021; (8): 82-84.

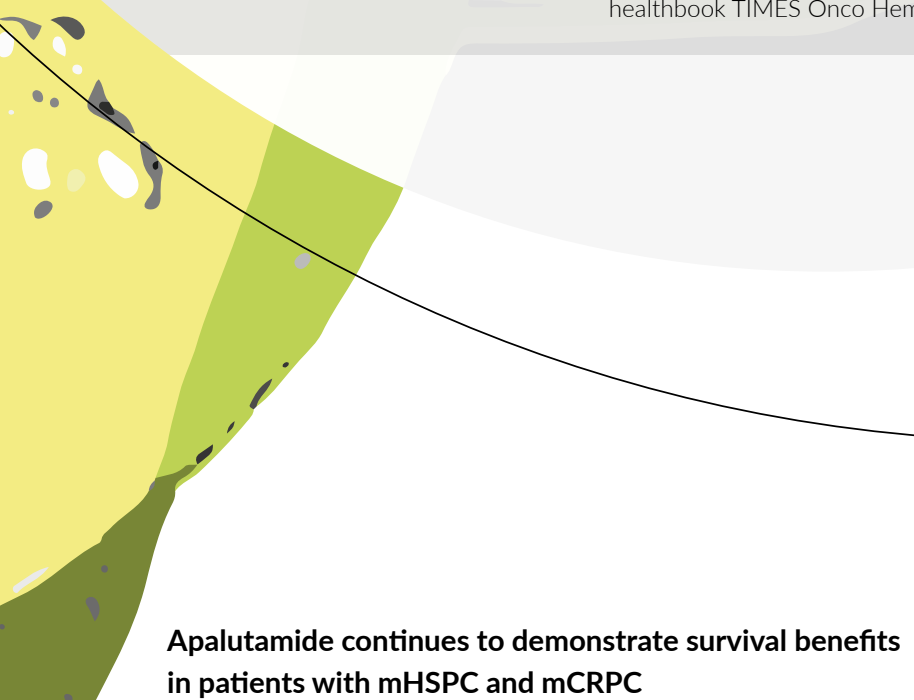

At ASCO Genitourinary (GU) Cancers Symposium 2021, Dr Kim Chi presented the final analysis of the phase III TITAN trial ${ }^{1}$, which investigated the efficacy and safety of apalutamide in combination with androgen-deprivation therapy (ADT) versus placebo in patients with metastatic, hormone-sensitive prostate cancer $(\mathrm{mHSPC})^{2}$. After a median follow-up of 44 months, apalutamide plus ADT reduced the risk of death by 35\% (HR: 0.65 [95\% Cl: 0.53-0.79]; $\mathrm{p}<0.0001$ ). After adjusting for a crossover ( 40\%), the HR improved to 0.52 [95\% Cl: 0.42-0.64]; $\mathrm{p}<0.0001$ ), which translated into a $48 \%$-reduction in the risk of death with apalutamide. This overall survival (OS) benefit was maintained across all prespecified subgroups, including disease volume, baseline lactate dehydrogenase (LDH) level and the presence of visceral disease. Results also showed that healthrelated quality of life (HRQoL) is maintained with apalutamide and somewhat improved compared with placebo after 2.5 years of treatment. There were no new safety signals. Cardiovascular events are also a side effect of ADT and augmented by secondgeneration AR inhibitors. This is a class-specific side effect compared with skin rash, which is rather specific for apalutamide but can be well-managed with the respective care. Taken together, apalutamide is another life-prolonging drug in the treatment armamentarium of $\mathrm{mHSPC}$ that facilitates a treatment selection based on patient characteristics and comorbidities.

Positive results were also obtained in the final analysis of the randomized, phase III ACIS trial, which showed that the addition of apalutamide to abiraterone acetate and prednisone (AAP) reduced the risk of radiographic progression or death by $30 \%$ in patients with chemotherapy-naïve metastatic castrationresistant prostate cancer (mCRPC). ${ }^{3}$ In this study, 982 patients underwent 1:1 randomization to receive AAP plus either apalutamide $(n=492)$ or placebo $(n=490)$. At a median followup of 54.8 months, the median radiographic progression-free survival (rPFS) was 24.0 months among patients receiving apalutamide plus AAP and 16.6 months among those receiving 


\section{Prostate Cancer Highlights from ASCO GU 2021}

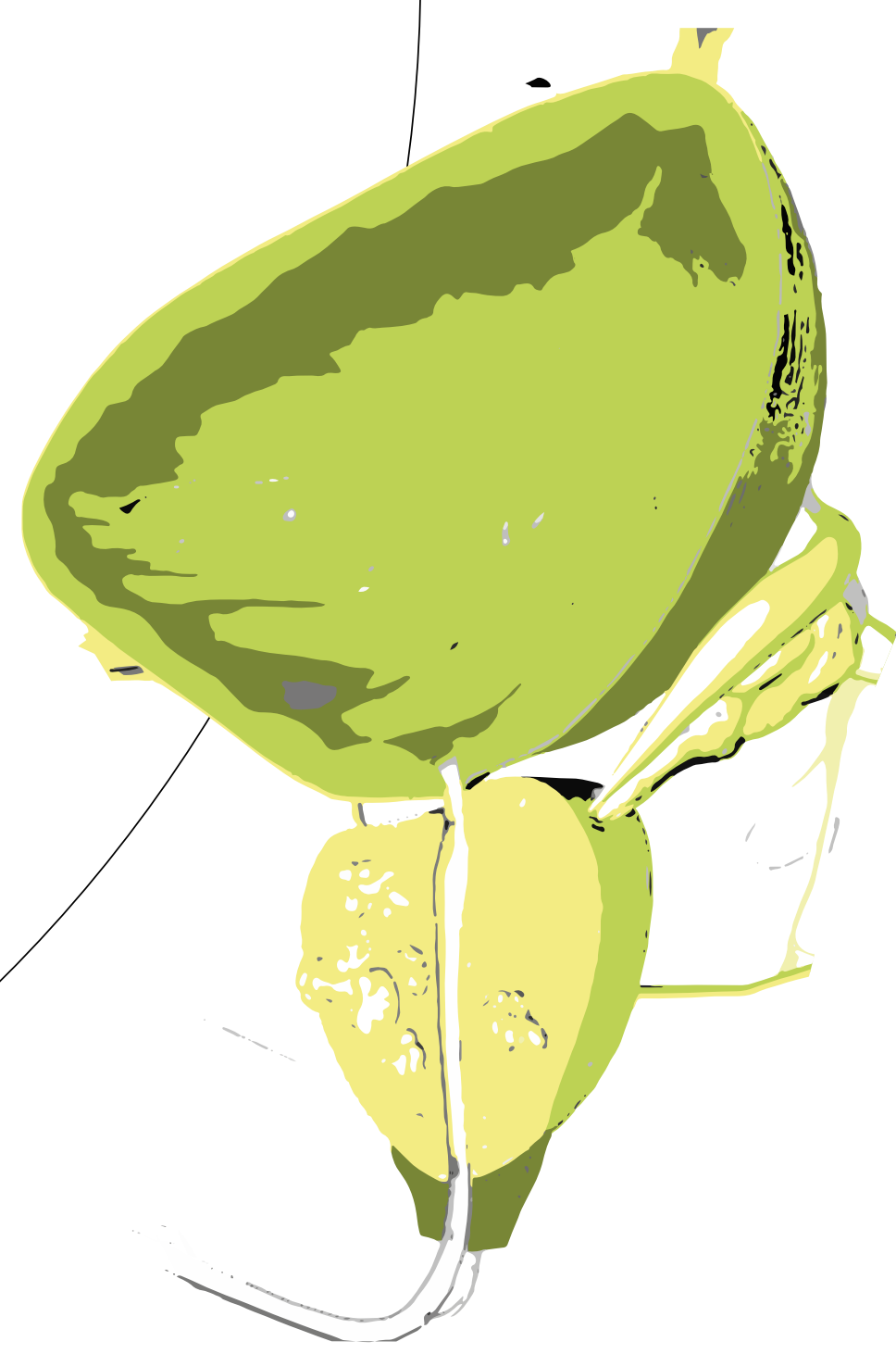

placebo plus AAP (HR: 0.70 [95\% Cl: 0.60-0.83]). The rPFS benefit with apalutamide plus AAP was sustained across prespecified subgroups, including patients aged $\geq 75$ years or the presence of visceral metastasis. Regarding secondary endpoints, there was no significant difference in OS, time to initiation of cytotoxic chemotherapy, pain progression, and chronic opioid use between the two treatment arms. However, there was a trend toward better OS outcomes with apalutamide plus AAP in selected prespecified biomarker subgroups, such as luminal histology or high androgen activity. Results further showed that $79.5 \%$ of patients in the apalutamide plus AAP arm had a prostate-specific antigen (PSA) decline of $\geq 50 \%$ compared with $72.9 \%$ in the control arm ( $p=0.015$ ), with $24.6 \%$ versus $19.2 \%$ of patients having undetectable PSA $(<0.2 \mathrm{ng} / \mathrm{mL})$ at any time during treatment $(p=0.040)$. In the final analysis, no new safety signals were reported. About two-thirds of patients treated with apalutamide experienced treatment-emergent adverse events (TEAEs), compared with $56.2 \%$ of placebo-treated patients. Grade 3-4 TEAEs that were more common in the apalutamidebased arm compared with the placebo-based arm included hypertension, fatigue and cardiac disorders.

\section{${ }^{177}$ Lu-PSMA-617 is a new class of effective therapy for patients} with mCRPC

Lutetium-177 (177Lu)-PSMA-617 (LuPSMA), a radiolabelled small molecule that binds with a high affinity to prostatespecific membrane antigen (PSMA), was used in countries like Germany, Austria or Australia (access granted by specialized clinical programs or individual permissions) over many years but until now, data from randomized trials concerning the efficacy and safety of this therapy were not available. At ASCO GU 2021, Prof. Michael Hofman presented the updated results of the randomized, phase II TheraP trial comparing LuPSMA and cabazitaxel $\left(20 \mathrm{mg} / \mathrm{m}^{2}\right)$ in 200 men with docetaxel-treated metastatic castration-resistant prostate cancer (mCRPC). ${ }^{4}$ Eligible patients had an Eastern Cooperative Oncology Group (ECOG) performance status (PS) of $0-2$ and PSA $\geq 20 \mathrm{ng} / \mathrm{ml}$. Of note, patients with low PSMA expression and FDG discordant disease were excluded from the study. The study met its primary endpoint as more patients receiving LUPSMA achieved a higher PSA response, defined by a $>50 \%$ reduction from baseline, than patients receiving cabazitaxel (66\% vs 37\%). LuPSMA versus cabazitaxel also improved both, PFS (HR: 0.63 [95\% Cl: 0.460.86 ]; $p=0028$ ) and objective response rate (ORR) (49\% vs $24 \%$ ). While grade 1-2 thrombocytopenia, dry mouth and dry eye were more common among LuPSMA-treated versus cabazitaxeltreated patients, LuPSMA was associated with fewer grade 3-4 adverse events (AEs). Surprisingly, cabazitaxel demonstrated a considerably lower rate of neutropenia (grade 1-2: $5 \%$; grade 3-4: 13\%) in comparison with our research, which showed that the rate of grade $\geq 3$ neutropenia ranges between $40 \%$ and $82 \%$ and is associated with improved outcome parameters such as PSA response rate, PFS and OS. Several patient-reported outcomes also favored LuPSMA. As stated by Prof. Hofman, the trial was relatively small and lacked OS data, a follow-up and the results of the VISION trial has thus been of great importance., ${ }^{5,6}$ Nevertheless, LuPSMA is an active treatment with a good safety profile, which might soon replace some of the current therapies utilized in clinical practice.

\section{Immune checkpoint inhibitors and prostate cancer: What is new?}

KEYNOTE-365 is a phase Ib/II study designed to investigate the safety and efficacy of pembrolizumab in combination with 4 different drug combinations in patients with mCRPC. $^{7}$ At ASCO GU 2021, Dr Leonard Appleman presented new efficacy and safety data from cohort $B$ receiving pembrolizumab plus docetaxel and prednisone, after an additional year of followup. ${ }^{8}$ This study included patients who did not respond to or were intolerant to $\geq 4$ weeks of abiraterone or enzalutamide in the prechemotherapy mCRPC state and whose disease progressed within 6 months of screening. At data cut-off, both confirmed and unconfirmed PSA response rates (PSA decrease $>50 \%$ ) were $43.7 \%$, while the median radiographic PFS (rPFS) was 8.5 months and the median OS was 20.2 months. Although the authors claimed that survival outcomes and response rates have been encouraging, these results are comparable with those from the TAX $327^{9}$, SWOG 99-16 ${ }^{10}$ and FIRSTANA ${ }^{11}$ trials, which demonstrated a median OS of 20-25 months, exceeding 30 months in the subgroup of patients with severe neutropenia. 
In my opinion, these results do not confirm the superiority of this pembrolizumab-based regimen over chemotherapy alone. A similar conclusion can be drawn when interpreting data from arm B of the phase II CHECKMATE 9KD trial, which assessed the efficacy and safety of nivolumab in combination with rucaparib, docetaxel and prednisone in patients with mCRPC. ${ }^{12}$ The median PSA change from baseline for all patients $(n=81)$ was $54.6 \%$ and more than half of the patients were responders. Notably, slightly better activity was observed in patients without prior exposure to novel antiandrogen therapy (NAT). At a median follow-up of 15.2 months, the median rPFS was 9.0 months and the median OS was 18.2 months. As these results were similar to the KEYNOTE-365 trial, the question remains whether this treatment combination is superior to chemotherapy alone and how to identify the patient achieving the greatest benefit from the addition of $\mathrm{ICl}$.

\section{SARS-CoV-2 and prostate cancer: Has ADT impacted COVID-19 outcomes?}

A significantly higher number of men were affected in the first wave of coronavirus disease-2019 (COVID-19). After the discovery that transmembrane protease serine 2 (TMPRSS2) promotes the entry of severe acute respiratory syndromerelated coronavirus (SARS-CoV-2) into the host cells, specialists in the field of prostate cancer discussed whether ADT can down-regulate the TMPRSS2 and decrease the probability of infection or improve the clinical outcomes. At ASCO GU 2021, Dr Daniel Kwon reported no association between the use of ADT and SARS-CoV-2 infection (odds ratio [OR]: 1.18) in a study of a diverse patient population at the University of California Health System medical centers and hospitals. ${ }^{13}$ However, racial or ethnic disparities were observed as African-American and Hispanic patients had a twice as high OR for COVID-19 infection in comparison with Whites.

Similar results were obtained in a study from the CCC19 Prostate Cancer Working Group, which provided evidence that ADT was not associated with improved COVID-19 outcomes. ${ }^{14}$ In addition, a high overall mortality rate of $15 \%$, irrespective of the receipt of ADT, was reported. The study also indicated that hydroxychloroquine (HCQ) and remdesivir led to significantly worse outcomes in terms of disease severity (adjusted OR:
8.21 and 5.61), while HCQ considerably increased 30-day mortality (adjusted OR: 5.15). However, these results might be biased as HCQ was most likely given to the extremely critical patients; the high mortality rate might also be related to the side effects of the drug. Furthermore, in a retrospective study, the ADT use in patients with prostate cancer prior to COVID-19 diagnosis did not influence the severity of infection as defined by hospitalization, oxygen utilization, and death. ${ }^{15}$ A subgroup analysis also demonstrated that age is the most important predictor of OS ( $\geq 70$ years vs $<70$ years; HR: 3.65 ). Again, no difference in the survival curves was observed in patients with or without ADT.

\section{Role of biomarkers in prostate cancer}

A retrospective analysis of the phase III PROSPER trial, which evaluated the safety and efficacy of enzalutamide versus placebo in patients with nonmetastatic CRPC, showed the importance of the depth of response to enzalutamide treatment. ${ }^{16}$ About $85 \%$ of men receiving enzalutamide had a PSA decline of $\geq 50 \%$; of these, $38 \%$ of patients achieved PSA reduction $\geq 90 \%$ with actual nadir $<0.2 \mathrm{ng} / \mathrm{mL}$, and $27 \%$ achieved PSA reduction $\geq 90 \%$ with actual nadir $\geq 0.2 \mathrm{ng} / \mathrm{mL}$. Among enzalutamide-treated patients, there was a correlation between increased depth of PSA decline and improved both, OS and metastasis-free survival (MFS). These results suggest that we need more dynamic biomarkers that could help us to adjust treatment during the therapy.

Another study evaluated biomarker associations with rPFS' $^{17}$ from the phase III IPATential150 trial, which assessed the efficacy and safety of ipatasertib plus abiraterone versus abiraterone alone in patients with treatment-naïve $\mathrm{MCRPC}^{18}$. Previous data demonstrated an improved rPFS with the combination regimen in the intention-to-treat population as well as in patients with tumors with PTEN loss as assessed by immunohistochemistry (IHC). In the present exploratory analysis, among patients evaluable also for next-generation sequencing (NGS) analysis, there was a good concordance between PTEN loss by IHC and NGS. Notably, stratifying patients by PTEN loss determined by NGS confirmed the rPFS benefit with ipatasertib plus abiraterone, showing that survival outcomes can be significantly improved by the appropriate selection of biomarkers and the most appropriate method for their determination.
1. Chi KN et al. Final analysis results from TITAN: A phase III study of apalutamide (APA) versus placebo (PBO) in patients (pts) with metastatic castration-sensitive prostate cancer
(mCSPC) receiving androgen deprivation therapy (ADT). ASCO (mCSPC) receiving androgen deprivation therapy (ADT). ASCO Oral presentation 11

2. Chi KN et al. Apalutamide for Metastatic, CastrationSensitive Prostate Cancer. N Engl J Med. 2019; 381(1): 13-24 3. Rathkopf DE et al. Final results from ACIS, a randomized placebo (PBO)-controlled double-blind phase 3 study of apalutamide (APA) and abiraterone acetate plus prednisone (AAP) versus AAP in patients (pts) with chemo-naive metastatic castration-resistant prostate cancer (mCRPC). February 2021. Oral presentation 9. 4. Hofman MS et al. 177Lu-PSMA-617 (LuPSMA) versus cabazitaxel in metastatic castration-resistant prostate cancer cabazitaxel in metastatic castration-resistant prostate cance
(mCRPC) progressing after docetaxel: Updated results including progression-free survival (PFS) and patient-reported outcomes (PROs) (TheraP ANZUP 1603). ASCO Genitourinary 5. Sartor AO et al. VISION: An international, prospective, 5. Sartor AO multicenter, randomized phase III study of 177L PSMA-617 in the treatment of patients with progressive PSMA-positive metastatic castration-resistant prostate cance (mCRPC). J Clin Oncol. 2020; 38(6_suppl): TPS259.

6. Novartis announces positive result of phase III stud with radioligand therapy 177Lu-PSMA-617 in patients with advanced prostate cancer. Novartis March 2021 [Accessed April 2021]. Available from: https://www.novartis.com/news/ media-releases/novartis-announces-positive-result-phaseiii-study-radioligand-therapy-177lu-psma-617-patients advanced-prostate-cancer.

7. Kolinsky MP et al. KEYNOTE-365 cohort B updated results: Pembrolizumab (pembro) plus docetaxel and prednisone in abiraterone (abi) or enzalutamide (enza)-pretreated patients (pts) with metastatic castrate-resistant prostate cancer (mCRPC). J Clin Oncol. 2020; 38(6_suppl): 103.

8. Appleman $\sqcup$ et al. KEYNOTE-365 cohort B: Pembrolizumab (pembro) plus docetaxel and prednisone in abiraterone (abi) or enzalutamide (enza)-pretreated patients with metastatic castration-resistant prostate cancer (mCRPC)-New data after (15-17 folow up. AscO Genitourinary Cancers 9. Berthold DR et a Mitorthold DR et al. Docetaxel plus Prednisone or Updated Survival in the TAX 327 Study. J Clin Oncol. 2008; 26(2): $242-5$

10. Petrylak DP et al. Docetaxel and estramustine compared with mitoxantrone and prednisone for advanced refractory prostate cancer. N Eng J J Med. 2004: 351(15): 1513-20 11. Oudard S et al. Cabazitaxel Versus Docetaxel As First-Lin Therapy for Patients With Metastatic Castration-Resistant Prostate Cancer: A Randomized Phase III Trial-FIRSTANA. Clin Oncol. 2017; 35(28): 3189-97.

12. Fizazi K et al. CheckMate 9KD Arm B final analysis: Efficacy and safety of nivolumab plus docetaxel for chemotherapy-naive metastatic castration-resistant prostate cancer. ASCO Ge nitourinary Cancers Symposium; 15-17 February 2021. Ora presentation 12
13. Kwon $\mathrm{D}$ et al. Androgen deprivation therapy and risk of SARS-CoV-2 infection in men with prostate cancer: A University of California (UC) Health System registry study. ASCO Genitourinary Cancers Symposium; 15-17 February

2021. Oral presentation 37.
14. Tucker MD et al. Severe-COVID-19 and mortality among patients (pts) with prostate cancer (PCa) receiving androgen deprivation therapy (ADT). ASCO Genitourinary Cancers Symposium; 15-17 February 2021. Poster presentation 39. 15. Patel VG et al. The role of androgen deprivation therapy on the clinical course of COVID-19 infection in men with prostate cancer. ASCO Genitourinary Cancers Symposium; 15-17

16. Hussain MHA et al. Overall survival (OS) and metastasis(PSA) decline in the by depth of prostate-specific antigen (PSA) decline in the phase III PROSPER trial of men with nonmetastatic castration-resistant prostate cancer (nmCRPC) treated with enzalutamide (ENZA). ASCO Genitourinary
Cancers Symposium; 15-17 February 2021. Poster presentation 94.

17. De Bono JS et al. I3K/AKT pathway biomarkers analysis from the phase III IPATential150 trial of ipatasertib plus abiraterone in metastatic castration-resistant prostate cancer. ASCO Genitourinary Cancers Symposium; 15-17 February 2021. Oral presentation 13.

18. de Bono J et al. 2020. IPATential150: Phase III study of ipatasertib (ipat) plus abiraterone (abi) vs placebo (pbo) plus abi in metastatic castration-resistant prostate cancer (mCRPC). ESMO Virtual Congress 2020; 19-21 September 2020. Oral presentation LBA4. 